\title{
Anisotropic Pair Correlations and Structure Factors of Confined Hard-Sphere Fluids: An Experimental and Theoretical Study
}

\author{
K. Nygård, ${ }^{1, *}$ R. Kjellander, ${ }^{2}$ S. Sarman, ${ }^{3}$ S. Chodankar, ${ }^{1}$ E. Perret,,${ }^{1, \dagger}$ J. Buitenhuis, ${ }^{4}$ and J. F. van der Veen ${ }^{1,5}$ \\ ${ }^{1}$ Research Department of Synchrotron Radiation and Nanotechnology, Paul Scherrer Institut, 5232 Villigen PSI, Switzerland \\ ${ }^{2}$ Department of Chemistry, University of Gothenburg, SE-412 96 Gothenburg, Sweden \\ ${ }^{3}$ Department of Materials and Environmental Chemistry, Stockholm University, SE-106 91 Stockholm, Sweden \\ ${ }^{4}$ Institute of Complex Systems, Forschungszentrum Jülich, 52425 Jülich, Germany \\ ${ }^{5}$ ETH Zürich, 8093 Zürich, Switzerland \\ (Received 6 October 2011; published 18 January 2012)
}

\begin{abstract}
We address the fundamental question: how are pair correlations and structure factors of hard-sphere fluids affected by confinement between hard planar walls at close distance? For this purpose, we combine $\mathrm{x}$-ray scattering from colloid-filled nanofluidic channel arrays and first-principles inhomogeneous liquidstate theory within the anisotropic Percus-Yevick approximation. The experimental and theoretical data are in remarkable agreement at the pair-correlation level, providing the first quantitative experimental verification of the theoretically predicted confinement-induced anisotropy of the pair-correlation functions for the fluid. The description of confined fluids at this level provides, in the general case, important insights into the mechanisms of particle-particle interactions in dense fluids under confinement.
\end{abstract}

DOI: 10.1103/PhysRevLett.108.037802

PACS numbers: 61.20.Gy, 61.05.cf, 68.08. $-\mathrm{p}$

Physical confinement of a fluid to a few times the particle diameter is known to induce ordering of the fluid along the confinement direction [1,2], and the ensuing layered density profile has been directly observed experimentally in a few cases (see, e.g., Ref. [3] and references therein). The confinement also affects other properties of the fluid, such as the diffusion of its constituents [4], freezing transitions [5], capillary condensation [6], and glass transition [7], and is therefore of both scientific and technological importance. However, the properties of the confined fluid cannot be understood simply in terms of its average density profile along the confining direction; rather, one has to consider the complex interplay between the density profile, i.e., the singlet correlation function, and the so-called local structure, i.e., the pair-correlation function.

The pair correlations of bulk fluids composed of spherical particles are isotropic. In contrast, inhomogeneous liquid-state theory has already a long time ago predicted that the pair correlations of inhomogeneous fluids are anisotropic [8,9], and the pair correlations of confined fluids have even been explicitly calculated in some cases; see, e.g., Refs. [10-14]. However, to date no quantitative experimental verification of these predictions has been reported. Recently, some of us reported an approach based on x-ray scattering from colloid-filled nanofluidic channel arrays, which allows us to address this question [15]. Within this scheme, we can reconstruct the density profile of the confined fluid in a model-independent manner by combining x-ray diffraction and phase-retrieval techniques. This approach has previously been used to study, e.g., trapping of colloidal monolayers near charged walls [16]. Moreover, owing to the 1000 -fold increase in scattering volume, as compared to using a single channel for confinement, we can simultaneously probe the pair correlations of the fluid, using the diffuse x-ray scattering originating from the short-range fluid density variations.

In this Letter, we combine the aforementioned $\mathrm{x}$-ray scattering from colloid-filled channel arrays [15] with first-principles inhomogeneous liquid-state theory within the anisotropic Percus-Yevick approximation $[9,11]$ in order to study the singlet and pair correlations of the most fundamental confined fluid: the hard-sphere fluid confined between hard planar walls. The experiment and theory are in excellent agreement at the pair-correlation level, thereby providing the first quantitative experimental verification of the theoretically predicted anisotropic pair correlations of confined fluids. The simultaneous experimental and theoretical description of confined fluids at this level will allow detailed studies on the interaction mechanisms in confined fluids. In particular, this approach will be of importance in studies on confined fluids exhibiting more complex interaction potentials, which are not accurately known a priori.

The overwhelming majority of theoretical studies on the structure of confined fluids, apart from simulations, have used simpler and in general less accurate kinds of approximations than the integral equation theory used in this work. They are formulated on the singlet density profile level and do not consider the anisotropic pair distributions. In simulations one can in principle evaluate pair distributions from the particle configurations, but to obtain a reasonable statistical accuracy in inhomogeneous fluids one would need extremely long runs, which makes this approach hardly feasible. The pair distributions can alternatively be evaluated pointwise in simulations by using the Widom insertion technique, which is feasible provided the 
fluid is not too dense. This has been done for inhomogeneous electrolytes and compared to the kind of integral equation techniques used here [17]. The integral equation approach can be more than a hundred thousand times more efficient in CPU time than the simulations for the corresponding amount of pair distribution data of the inhomogeneous system and give results of virtually equal accuracy in the cases that have been compared.

The x-ray scattering approach of Ref. [15] probes the pair correlations of the confined fluid in terms of its anisotropic structure factor,

$$
S(\mathbf{q})=1+\frac{1}{N} \iint n(\mathbf{r}) n\left(\mathbf{r}^{\prime}\right) h\left(\mathbf{r}, \mathbf{r}^{\prime}\right) e^{i \mathbf{q} \cdot\left(\mathbf{r}^{\prime}-\mathbf{r}\right)} d \mathbf{r} d \mathbf{r}^{\prime} .
$$

Here $\mathbf{q}$ denotes the scattering vector, $h\left(\mathbf{r}, \mathbf{r}^{\prime}\right)=g\left(\mathbf{r}, \mathbf{r}^{\prime}\right)-1$ the total correlation function, $g\left(\mathbf{r}, \mathbf{r}^{\prime}\right)$ the pair distribution function, $n(\mathbf{r})$ the number density profile, and $N$ the total number of particles, while the integration is carried out over particle positions $\mathbf{r}$ and $\mathbf{r}^{\prime}$. In contrast to bulk fluids, the lack of translational invariance along the confining direction hinders the direct determination of the experimental $h\left(\mathbf{r}, \mathbf{r}^{\prime}\right)$ by Fourier inversion. Instead, we determine $h\left(\mathbf{r}, \mathbf{r}^{\prime}\right)$ and $n(\mathbf{r})$ theoretically and compare the results to the experimental $S(\mathbf{q})$ using Eq. (1).

Following the approach outlined in Refs. [11,18], we determine the theoretical $h\left(\mathbf{r}, \mathbf{r}^{\prime}\right)$ and $n(\mathbf{r})$ by solving two exact integral equations: the inhomogeneous OrnsteinZernike equation,

$$
h\left(\mathbf{r}, \mathbf{r}^{\prime}\right)=c\left(\mathbf{r}, \mathbf{r}^{\prime}\right)+\int h\left(\mathbf{r}, \mathbf{r}^{\prime \prime}\right) n\left(\mathbf{r}^{\prime \prime}\right) c\left(\mathbf{r}^{\prime \prime}, \mathbf{r}^{\prime}\right) d \mathbf{r}^{\prime \prime},
$$

and the Lovett-Mou-Buff-Wertheim equation,

$$
\nabla[\log n(\mathbf{r})+\beta v(\mathbf{r})]=-\beta \int n\left(\mathbf{r}^{\prime}\right) h\left(\mathbf{r}, \mathbf{r}^{\prime}\right) \nabla^{\prime} v\left(\mathbf{r}^{\prime}\right) d \mathbf{r}^{\prime} .
$$

Here $c\left(\mathbf{r}, \mathbf{r}^{\prime}\right)$ is the direct correlation function, $v(\mathbf{r})$ the particle-wall interaction potential, and $\beta=\left(k_{B} T\right)^{-1}$ with $k_{B}$ Boltzmann's constant and $T$ the absolute temperature. Throughout this study we have made use of the PercusYevick (PY) closure for the anisotropic pair correlations, $c\left(\mathbf{r}, \mathbf{r}^{\prime}\right)=g\left(\mathbf{r}, \mathbf{r}^{\prime}\right)-y\left(\mathbf{r}, \mathbf{r}^{\prime}\right)$, where $y\left(\mathbf{r}, \mathbf{r}^{\prime}\right)$ is the cavity function that satisfies $g\left(\mathbf{r}, \mathbf{r}^{\prime}\right)=y\left(\mathbf{r}, \mathbf{r}^{\prime}\right) \exp \left[-\beta u\left(\mathbf{r}, \mathbf{r}^{\prime}\right)\right]$ and $u\left(\mathbf{r}, \mathbf{r}^{\prime}\right)$ is the particle-particle interaction potential (note that there is a misprint in the PY closure Eq. (6) of Ref. [18]). Hard particle-particle and particle-wall interaction potentials were used. We kept the fluid in equilibrium with a bulk reservoir following Ref. [18], but see [19]. The pair correlations and the density profile were determined self-consistently in an iterative manner. Detailed plots of the theoretical pair distribution functions for hard spheres between hard walls can be found in Ref. [11].

The experiment was carried out at the cSAXS beam line of the Swiss Light Source. The incident $x$ rays impinged normal to the nanofluidic channel array and we collected the diffracted and diffusely scattered $\mathrm{x}$ rays simultaneously
$7 \mathrm{~m}$ behind the sample using the single-photon-counting PILATUS 2M pixel detector [20]. We used an X-ray wavelength of $\lambda=0.10 \mathrm{~nm}$ and a beam size of $0.2 \times 0.2 \mathrm{~mm}^{2}$ at the sample position. The incident $\mathrm{x}$-ray beam was focused onto the detector plane in order to maximize the angular resolution and an evacuated flight tube was used between the sample and the detector to minimize parasitic scattering. We could reliably determine the diffraction efficiencies up to 50 diffraction orders, which allows a reconstruction of the density profile of the fluid with a real-space sampling interval of $4.0 \mathrm{~nm}$. We have verified the model-independent density reconstruction using both iterative [21] and noniterative [22] phase-retrieval schemes. The anisotropic structure factor of the confined fluid was obtained by subtracting from the measured scattering pattern the scattering from the channel array [23] and the diffuse scattering contribution from the fluid reservoir on top of the cavity array. The experiment was carried out at a temperature of $T=297 \mathrm{~K}$.

The fluid consisted of octadecyl-grafted spherical silica particles dispersed in toluene. At the temperature of the present study, the colloidal suspension acts like a hardsphere fluid, as has been shown in Ref. [24] for the present batch of the suspension. This also provides support for using hard-sphere interactions in the theoretical calculations. The silica particles had an average diameter of $\sigma_{0}=64.6 \mathrm{~nm}$ and a polydispersity of $\Delta \sigma_{0} / \sigma_{0}=0.124$. Considering also the $2.3 \mathrm{~nm}$ thick octadecyl layer grafted on the particles, we obtain an average particle diameter of $\sigma=69.2 \mathrm{~nm}$. The bulk density was $n_{b}=0.75 \sigma^{-3}$, corresponding to a bulk particle volume fraction $\phi_{b}=0.39$. Details on the synthesis and characterization of the colloidal suspension can be found elsewhere [24].

For confinement we used silicon nanofluidic channel arrays with the following parameters: a period of $400 \mathrm{~nm}$, a depth of $1.8 \mu \mathrm{m}$, and a total area of $0.5 \times 0.5 \mathrm{~mm}^{2}$ per channel array. The width of the confining channel was systematically varied in the range $w=170-215 \mathrm{~nm}$, corresponding to wall-wall distances in the range $w \approx$ $2.45 \sigma-3.10 \sigma$. The confining walls were structureless on the length scales relevant for the fluid, which is a prerequisite for observing confinement-induced fluid ordering. Details on the fabrication of the channel arrays have been published elsewhere [22].

First we consider the density profile of the confined fluid for selected channel widths. Following Ref. [21], we present the experimental data in Fig. 1 as the local volume fraction $C(z)$ occupied by the spheres, with $z$ being the position across the confining channel. The corresponding theoretical data are given by $C(z)=\left(\pi \sigma^{3} / 6\right) n(z) \otimes P(z)$, with $\otimes$ denoting the convolution operator and $P(z)$ the projection of the particles' spherical shape (see, e.g., Ref. [25]). In general, the agreement between experimental and theoretical density profiles is very good, with both experimental and theoretical data exhibiting a structural 


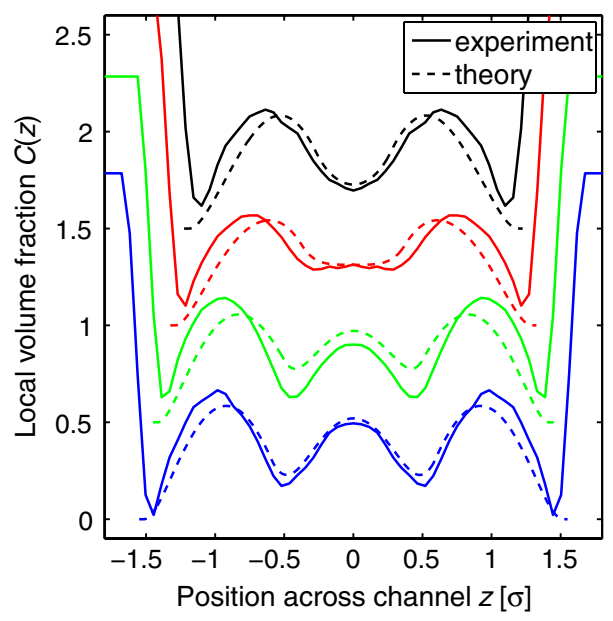

FIG. 1 (color online). Experimental (solid lines) and theoretical (dashed lines) local volume fractions $C(z)$ across the confining channel. The channel widths are, from top to bottom, $w \approx 2.45 \sigma, 2.65 \sigma, 2.90 \sigma$, and $3.10 \sigma$, and the profiles are vertically offset by multiples of 0.5 for clarity. The nearly vertical lines in the experimental data depict the confining walls.

transition from two to three particle layers with increasing channel width. We note, however, that subtle details in the number density profile $n(z)$ cannot be resolved in the local volume fraction $C(z)$, because of the smearing with $P(z)$.

A minor systematic shift in peak positions between the experimental and theoretical $C(z)$ is observed in Fig. 1. We have determined the channel widths $w$ independently using both x-ray diffraction and scanning electron microscopy, thereby ruling out the possibility of a systematic error in the experimentally determined $w$. Also, dynamical diffraction effects, due to a nonplanar $\mathrm{x}$-ray wave field inside the channels, can be ruled out as a source of the shift [26]. Instead, two differences between the experimental and theoretical systems should be mentioned as possible sources of the shift. First, in contrast to the experimental system, the theoretical profiles do not include size polydispersity of the particles, which is expected to induce local size segregation in the density profile [27]. Second, whereas the theoretical profiles are determined for perfectly parallel walls, the fabrication process induces a minor variation in the experimental channel width of $\Delta w \approx \pm 0.05 \sigma$ along the depth of the channel.

Next we turn to the effect of confinement on the pair correlations. This is presented in Fig. 2 as the experimental and theoretical anisotropic structure factors $S\left(q_{\perp}, q_{\|}\right)$for the different channel widths of Fig. 1, with $q_{\perp}$ and $q_{\|}$ denoting the out-of-plane and in-plane components of the scattering vector, respectively. In order to facilitate the comparison between experiment and theory, we have determined the experimental $S(\mathbf{q})$ within the monodisperse approximation, which is a reasonable approximation for the present colloidal suspension [24]. The agreement between experimental and theoretical data is striking. Not only is the theory accurately describing the channel-width dependence of both the positions and amplitudes of the primary (first) peaks in $S(\mathbf{q})$, but also correctly predicts the lobes and secondary maxima at larger scattering vectors. Keeping in mind that there are no fitting parameters in the calculation, the agreement in Fig. 2 clearly demonstrates the accuracy and predictive power of the adopted
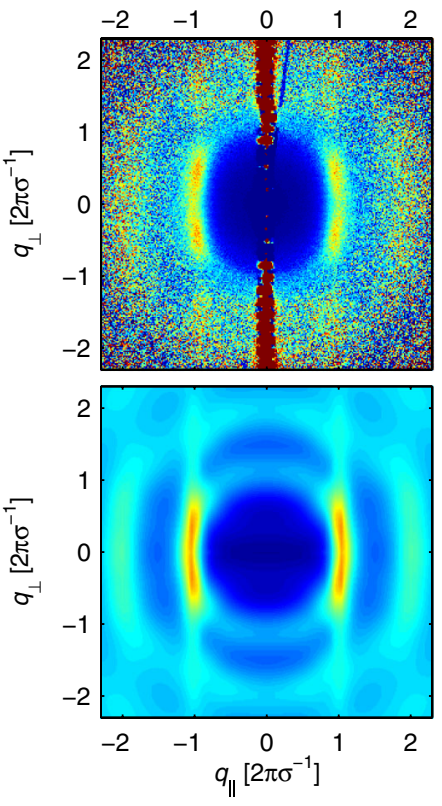
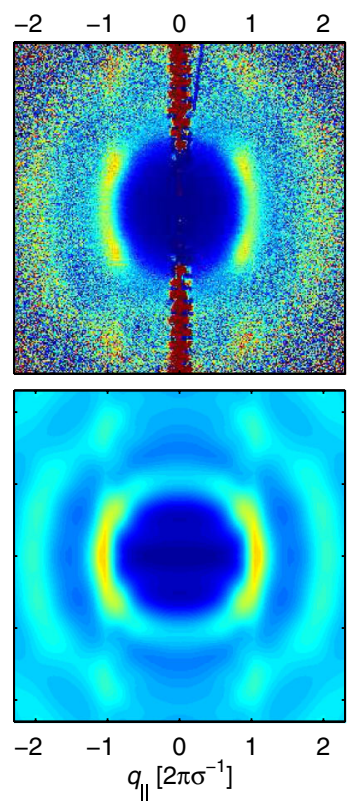
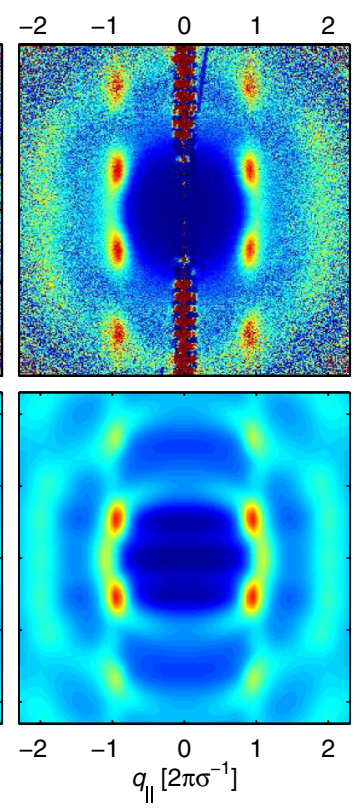

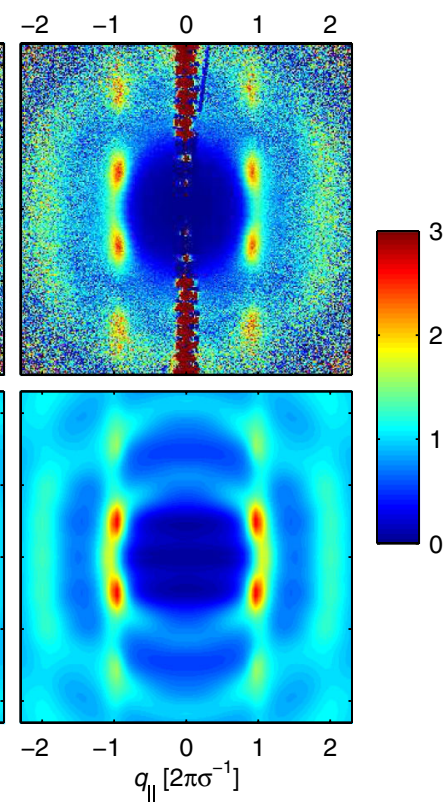

FIG. 2 (color online). Experimental (top) and theoretical (bottom) anisotropic structure factors $S\left(q_{\perp}, q_{\|}\right)$. The channel widths are, from left to right, $w \approx 2.45 \sigma, 2.65 \sigma, 2.90 \sigma$, and $3.10 \sigma$. The feature at $q_{\|}=0$ in the experimental data is diffraction from the fluidfilled nanofluidic channel array, which was used to obtain the density profiles shown in Fig. 1. 
theoretical scheme. Nevertheless, since the inaccuracy in the experimental $S(\mathbf{q})$ increases for larger scattering vectors, we will in the remainder of this Letter concentrate on the primary peaks in $S(\mathbf{q})$.

During the structural transition from two to three particle layers, we observe the emergence (or strengthening) and disappearance (or weakening) of distinct peaks in $S(\mathbf{q})$. Qualitatively this effect can be explained by the particles predominantly residing in the hollow sites of the other layers [15]. During the change from two to three layers, the packing of spheres becomes less efficient than when the layers are well formed; i.e., a kind of "frustrated" fluid structure forms at intermediate slit widths. All these effects can be clearly seen in the theoretical pair distribution plots of Ref. [11] at somewhat larger slit widths. In order to obtain a quantitative real-space picture of the mechanisms behind the layer formation, we would need to further study in detail the theoretical $n(z) g\left(\mathbf{r}, \mathbf{r}^{\prime}\right)$, i.e., the density at coordinate $\mathbf{r}$ in the neighborhood of a particle at $\mathbf{r}^{\prime}$ for different positions of the latter in the confining channel. Such an elaborate analysis is beyond the scope of the present study and will be presented in a separate publication.

A qualitative discrepancy between the experimental and theoretical data is observed in Fig. 2 for the channel width of $w \approx 2.65 \sigma$. The theoretical $S\left(q_{\perp}, q_{\|}\right)$exhibits maxima for $q_{\perp}=0$ at $q_{\|} \approx \pm 2 \pi \sigma^{-1}$, which are not present in the experimental data (see the color version of the figure). In fact, these in-plane peaks in the theoretical $S\left(q_{\perp}, q_{\|}\right)$are clearly observed only in a very narrow range of channel widths, $w \approx 2.60 \sigma-2.70 \sigma$. These effects are associated with the frustrated fluid structure mentioned above. Considering the polydispersity of the experimental system and the variation in slit width of $\approx \pm 0.05 \sigma$ as described above, the packing conditions may not have been exactly the same in the experiment and the theoretical calculations. Again, a detailed analysis of this phenomenon is beyond the scope of this Letter and will be published elsewhere.

Given the strong anisotropies observed in the structure factors of Fig. 2, one might question whether the system is still in the fluid phase. Indeed, the bulk number density $n_{b}=0.75 \sigma^{-3}$ is close to the onset of solid-fluid coexistence, which for monodisperse hard-sphere particles is $n_{\mathrm{sf}} \geq 0.78 \sigma^{-3}$ for the channel widths of our experiment [28]. However, the primary maxima of the experimental structure factor, $S\left(q_{\max }^{1 \mathrm{st}}\right)$, are below the Hansen-Verlet criterion for freezing [29], which is $S\left(q_{\max }^{1 \text { st }}\right) \approx 2.85$ for bulk fluids. Even more importantly, we have verified both experimentally and theoretically that the anisotropy is clearly observable also for smaller bulk concentrations (at least down to $n_{b}=0.38 \sigma^{-3}$ ), confirming that the observed effect is a fluid-phase phenomenon.

Next, we comment briefly on the generality of the observed confinement-induced features of the pair correlations in the fluid. The main features of the pair correlations of various simple liquids and complex fluids exhibiting short-ranged particle-particle interactions are governed by the excluded volume of the core region, which is contained in the hard-sphere fluid. Similarly, the main effects of short-ranged particle-wall interactions are given by the excluded volume at the interface, which is contained in the present system of a hard-sphere fluid between hard planar walls. In fact, theoretical work on confined Lennard-Jones fluids has provided anisotropic paircorrelation functions similar to those of confined hardsphere fluids [12]. Given these considerations, we argue that the large anisotropy of the pair correlations observed both experimentally and theoretically in this study is an intrinsic property of a wide class of dense simple liquids and complex fluids in confinement. Consequently, we expect this property to have a large impact on many properties of these fluids, such as the diffusive dynamics and various phase transitions in confined geometries alluded to in the introduction.

Finally, we note that the simultaneous experimental and theoretical description of confined fluids at the paircorrelation level, as demonstrated here, provides a unique means to study particle-particle and particle-wall interactions in confined fluids. For the future, we foresee extending the present work to confined fluids exhibiting interaction potentials differing from those of the hardsphere hard-wall system studied here.

The experiment was carried out at the cSAXS beam line of the Swiss Light Source, Paul Scherrer Institut, Villigen, Switzerland. We thank the beam line staff for assistance. One of us (R. K.) gratefully acknowledges support from the Swedish Research council. The calculations in this work were supported by the Swedish National Infrastructure for Computing (SNIC 001-09-152) via PDC.

*Present address: Department of Chemistry, University of Gothenburg, Sweden. kim.nygard@chem.gu.se

†Present address: Argonne National Laboratory, Argonne, IL 60439, USA.

[1] J.-P. Hansen and I. R. McDonald, Theory of Simple Liquids (Academic Press, Amsterdam, 2006), 3rd ed.

[2] J. N. Israelachvili, Intermolecular and Surface Forces (Academic Press, London, 1991), 2nd ed.

[3] O. Bunk et al., Phys. Rev. E 75, 021501 (2007).

[4] J. Mittal, T. M. Truskett, J. R. Errington, and G. Hummer, Phys. Rev. Lett. 100, 145901 (2008).

[5] M. Schmidt and H. Löwen, Phys. Rev. Lett. 76, 4552 (1996).

[6] R. Evans and P. Tarazona, Phys. Rev. Lett. 52, 557 (1984).

[7] S. Lang, V. Botan, M. Oettel, D. Hajnal, T. Franosch, and R. Schilling, Phys. Rev. Lett. 105, 125701 (2010).

[8] J.K. Percus, in The Equilibrium Theory of Classical Fluids, edited by H.L. Frisch and J.L. Lebowitz (Benjamin, New York, 1964), pp. II.33-II.170. 
[9] S. Sokolowski, J. Chem. Phys. 73, 3507 (1980).

[10] R. Kjellander and S. Marčelja, J. Chem. Phys. 88, 7138 (1988).

[11] R. Kjellander and S. Sarman, J. Chem. Soc., Faraday Trans. 87, 1869 (1991).

[12] R. Kjellander and S. Sarman, Mol. Phys. 74, 665 (1991).

[13] B. Götzelmann and S. Dietrich, Phys. Rev. E 55, 2993 (1997).

[14] D. Henderson, S. Sokolowski, and D. Wasan, J. Stat. Phys. 89, 233 (1997).

[15] K. Nygård, D. K. Satapathy, J. Buitenhuis, E. Perret, O. Bunk, C. David, and J. F. van der Veen, Europhys. Lett. 86, 66001 (2009).

[16] D. K. Satapathy et al., Phys. Rev. Lett. 101, 136103 (2008).

[17] H. Greberg, R. Kjellander, and T. Åkesson, Mol. Phys. 87, 407 (1996); 92, 35 (1997).

[18] R. Kjellander and S. Sarman, Chem. Phys. Lett. 149, 102 (1988).

[19] The average number of particles per unit area in the slit as a function of the slit width was obtained from Eq. (12) of Ref. [18] cf. the procedure in Ref. [12]. This alternative is numerically more stable than the use of Eq. (13) of Ref. [18].
[20] P. Kraft et al., J. Synchrotron Radiat. 16, 368 (2009).

[21] O. Bunk, A. Diaz, F. Pfeiffer, C. David, B. Schmitt, D. K. Satapathy, and J. F. van der Veen, Acta Crystallogr. Sect. A 63, 306 (2007).

[22] K. Nygård, D. K. Satapathy, O. Bunk, E. Perret, J. Buitenhuis, C. David, and J.F. van der Veen, J. Appl. Crystallogr. 42, 1129 (2009).

[23] Prior to subtraction, the scattered intensities from the empty channel array were scaled so as to account for the reduced density contrast upon filling with the fluid. The feature at $q_{\|}=0$ in Fig. 2 is a remnant of the diffraction pattern.

[24] J. Kohlbrecher, J. Buitenhuis, G. Meier, and M.P. Lettinga, J. Chem. Phys. 125, 044715 (2006).

[25] M. C. Gerstenberg, J. S. Pedersen, and G. S. Smith, Phys. Rev. E 58, 8028 (1998).

[26] K. Nygård, D. K. Satapathy, O. Bunk, F. Pfeiffer, C. David, and J.F. van der Veen, Appl. Phys. Lett. 92, 214105 (2008).

[27] I. Pagonabarraga, M.E. Cates, and G. J. Ackland, Phys. Rev. Lett. 84, 911 (2000).

[28] A. Fortini and M. Dijkstra, J. Phys. Condens. Matter 18, L371 (2006).

[29] J.-P. Hansen and L. Verlet, Phys. Rev. 184, 151 (1969). 